\title{
Aplicación Móvil para la Administración de Variables Físicas en Ciclismo al Aire Libre
}

\author{
Andrea C. Alarcón-Aldana, Jorge Urrutia-Pinilla y Mauro Callejas-Cuervo \\ Universidad Pedagógica y Tecnológica de Colombia, Grupo Investigación en Software, Colombia. \\ (e-mail: acalarcon@gmail.com, georgeurru@gmail.com, mauro.callejas@uptc.edu.co)
}

Recibido Nov. 12, 2015; Aceptado Ene. 19, 2016; Versión final Feb. 25, 2016, Publicado Ago. 2016

\section{Resumen}

En el presente artículo se plantea la implementación de un software basado en computación móvil que permite capturar, controlar y monitorizar variables físicas relacionadas con la práctica del ciclismo. El objetivo es ofrecer una herramienta de acompañamiento y seguimiento tanto del rendimiento deportivo, como de aspectos inherentes a la salud propia del deportista o practicante. Uno de los aportes significativos de este trabajo es el desarrollo del módulo para gestionar datos como latitud, longitud, velocidad y altitud de una ruta programada, permitiendo la personalización de puntos de referencia en un recorrido específico, haciendo uso de tecnologías emergentes que facilitan el proceso.

Palabras claves: ciclismo; computación móvil; Android; entrenamiento; variables físicas

\section{Mobile Application for Physical Variables Management in Outdoor Cycling}

\begin{abstract}
In this article the implementation of a mobile computing based software to capture, control and monitor physical variables related to cycling is described. The purpose of this work is providing a tool support and monitoring not only athletic performance but also aspects inherent to health of the athlete or practitioner. One of the significant contributions of this work is the development of the module for managing data such as latitude, longitude, speed and altitude of a scheduled route, allowing customization of benchmarks on a specific route, using emerging technologies that facilitate the process.
\end{abstract}

Keywords: cycling; mobile computing; Android; training; physical variables 


\section{INTRODUCCIÓN}

Actualmente la práctica de actividad física ha crecido potencialmente, algunas personas que practican deporte lo asocian con lograr una mayor esperanza de vida y mejorar las condiciones de salud frente a aquellas que no lo hacen (Chi-Wai et al, 2011). La bicicleta es una de las principales y más populares herramientas usadas en la actividad física, que ha permitido adelantar estudios sobre la biomecánica del ciclismo y monitorización de variables, conducentes a la mejora en la ejecución de este deporte (Xu et al, 2015) además se ha impulsado el uso de dispositivos tecnológicos para el acompañamiento y la aplicación de buenas prácticas, lo que en algunos casos resulta costoso. Por otra parte, el rápido crecimiento de la popularidad de los video juegos y uso masivo de teléfonos inteligentes (Lu et al, 2013) representa una gran oportunidad de disponer de una solución tecnológica en la práctica y evolución del ciclismo mediante el uso de estos dispositivos, teniendo en cuenta su fácil acceso y los altos índices en su adopción en tareas cotidianas (Xie y Wu, 2012).

La práctica del ciclismo de manera recurrente genera grandes beneficios para la salud, reduce la posibilidad de desarrollar enfermedades cardiovasculares, diabetes e hipertensión y sustancialmente la posibilidad de sufrir de obesidad, entre otros (Dora y Phillips, 2015), lo que ha generado en los usuarios la necesidad de tener un control y monitorización permanente que permita constatar su evolución. En el mercado actual se encuentra productos que ofrecen dicha funcionalidad (Unocero, 2013), pero tienen como inconveniente el costo de venta, pues su funcionamiento se basa en gran parte en accesorios de Hardware desarrollados por compañías privadas. De igual manera se encuentra estudios en los cuales por medio del uso de teléfonos inteligentes y los sensores que estos incluyen, además de otras tecnologías, es posible monitorizar datos relevantes en la práctica del deporte tales como velocidad, inclinación, entre otros (Hintzy y Horvais, 2015; Hirose y Kitamura, 2015; Chi-Wai et al, 2011).

Por otro lado, Kikuchi et al (2012), presentan un simulador de ciclismo, el cual utilizando componentes electrónicos y mecánicos se encarga de medir la cadencia de pedaleo del usuario, controlar un mecanismo magnético que simula las pendientes de los mapas cargados en el ordenador principal y por medio de una pantalla gigante y el uso de gafas especiales permiten visualizar la ruta del recorrido en tres dimensiones (3D). A su vez, Moller et al (2012) presenta el desarrollo de GymSkill, un entrenador personal para el seguimiento y evaluación de la actividad física del usuario practicante del deporte por medio del uso de los sensores del teléfono inteligente en el cual se analizan la calidad de los movimientos y con el uso de la información recolectada brinda sugerencias para mejorar el rendimiento individual. Finalmente, Gaidos y Santos (2014) presentan un sistema para el seguimiento y formación de los ciclistas, que utilizando el Sistema de Posicionamiento Global (GPS) del teléfono inteligente, calculan la velocidad, altitud y dichos datos se envían a través de internet para su administración, y de igual manera han usado un dispositivo electrónico para la medición de la oximetría del usuario practicante del deporte.

El principal resultado de este trabajo fue el desarrollo del software que permite la administración de variables físicas, tales como velocidad, distancia recorrida, tiempo transcurrido, altura, así como las características de una ruta en una sesión de entrenamiento (punto de inicio y finalización, distancia total, puntos de referencia, inclinación, latitud, longitud y altitud). A diferencia de otros trabajos se programa de forma personalizada y posteriormente se usa en un plan de entrenamiento guiado por profesionales.

\section{EL MODELO}

El sistema presentado está enmarcado en el desarrollo del macro proyecto titulado, "Plataforma para la captura, control y monitorización de variables físicas y fisiológicas de ciclismo bajo techo y ciclismo al aire libre", coordinado por Grupo de Investigación en Software GIS, de la Universidad Pedagógica y Tecnológica de Colombia (UPTC), el cual tiene como finalidad la integración de la práctica de ciclismo en estos dos escenarios, mediante sesiones de entrenamiento conjuntas en el cual el ciclismo bajo techo simule las rutas trazadas en ciclismo al aire libre.

\section{Arquitectura del sistema general}

Actualmente cualquier proceso soportado en un software requiere de la identificación y propuesta de una arquitectura, tanto en el proceso de desarrollo como en el de mantenimiento, con el propósito de garantizar la evolución y acoplamiento de dicho sistema (Monroy et al, 2013), por esta razón se describe la arquitectura para el macro proyecto antes mencionado. La figura 1, muestra la distribución de los componentes de las dos modalidades de ciclismo, el cual incluye el desarrollo, para este caso, de los módulos relacionados con los numerales 2, 3, 5, 6, 8 y 10, parte izquierda de la figura. La aplicación móvil referente al ciclismo al aire libre, interactúa directamente con la aplicación servidor que contiene la información de las rutas de entrenamiento, sesiones de entrenamiento, información básica de los practicantes de ciclismo, entre otras. 


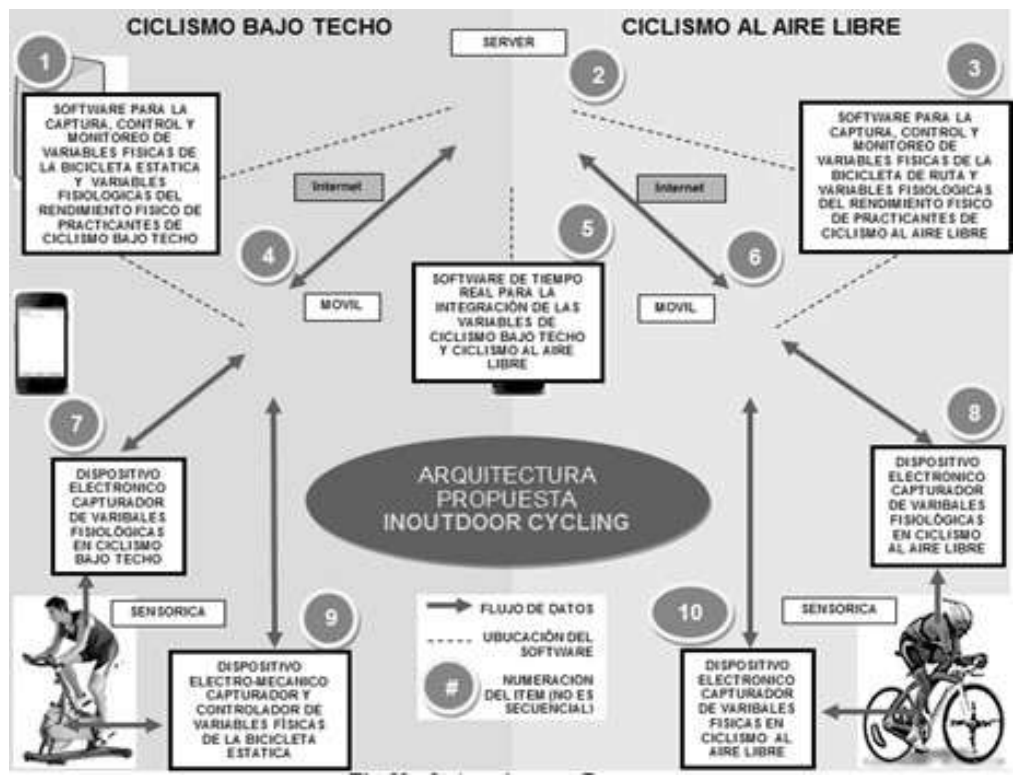

Fig. 1 Arquitectura de Componentes Propuesta para el macroproyecto del Grupo GIS

Para la comunicación entre el dispositivo móvil y el servidor de información se estableció como punto de transferencia de información digital, las redes GSM (Global System for Mobile comunications) y sus estándares GPRS (General Packet Radio Service) y EDGE (Enhanced Data Rates For Global Evolution), soportadas por el proveedor de servicio de telefonía celular, tal como se representa en la figura 2. Esta comunicación se realiza por medio de CSD (Circuit Switch Data) o HSCSD (High Speed Circuit Switched Data), esta última haciendo referencia a tecnologías $3 G \circ 4 G$, cuya disposición de servicio depende fundamentalmente de la empresa proveedora y de la localización geográfica del dispositivo móvil Android (Lizárraga et al, 2008).

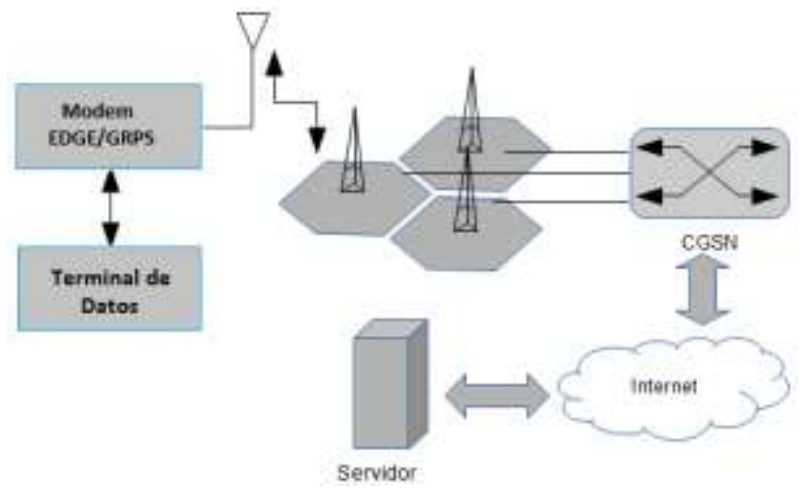

Fig. 2: Comunicación General Packet Radio Service, adaptado de (Lizárraga et al, 2008).

Por tal motivo y teniendo como principal referente la posible pérdida de información y rupturas de comunicación en lapsos de tiempo indefinidos, se establece la importancia de alojar información en el dispositivo móvil Android mediante el uso de SQLlite y su posterior sincronización de datos con el servidor principal como se puede ver en la figura 3.

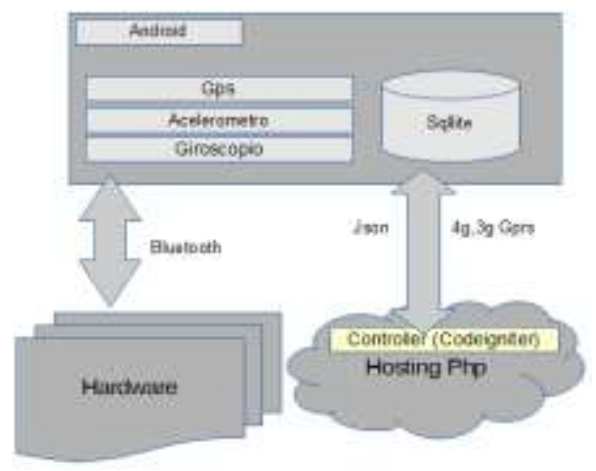

Fig. 3: Arquitectura de componentes de la aplicación móvil de ciclismo al aire libre 
La transmisión de paquetes se realiza por medio de componentes JSON, los cuales están diseñados para el intercambio de datos legibles, es fácil de utilizar y analizar, además ofrece ventajas respecto a la velocidad de transmisión que comparándolo con otros estándares como XML puede llegar a presentar velocidades 100 veces superiores (Nurseitov et al, 2015). Para su manipulación se usa Javascript el cual maneja la biblioteca DOM (Document Object Model). Para la implementación de la aplicación del servidor se usó el marco de desarrollo de aplicaciones Codeignither, que es un conjunto de herramientas que facilita la construcción de software para el lenguaje de programación PHP, y que se compone de un conjunto de bibliotecas de tareas comunes, una interfaz sencilla y una estructura lógica para acceder a dichas bibliotecas (EllisLab, 2015). Codeigniter, maneja el patrón de arquitectura de software MVC (Modelo Vista Controlador) como se puede ver en la Fig.4 cuyo objetivo es la separación de los datos, la lógica del negocio y la interfaz gráfica de usuario en componentes independientes que se comunican unos con otros para poder manipular la información de una manera clara y sencilla, facilitando el desarrollo y posterior mantenimiento de la aplicación (Caballé y Xhafa, 2007)

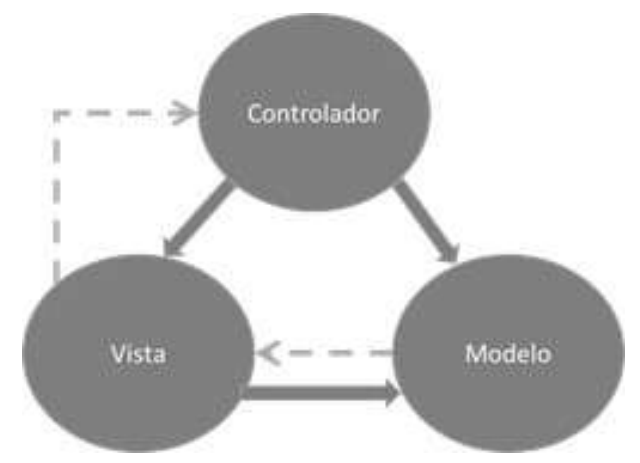

Fig. 4: patrón de arquitectura de software Modelo Vista Controlador

\section{Funcionalidades básicas del sistema}

El software permite el ingreso de datos básicos del practicante del deporte, tales como fecha de nacimiento, hábitos alimenticios, hábitos sociales (incluyendo entre otros aspectos si es o no fumador o si ingiere bebidas alcohólicas ocasionalmente o no), tipo y frecuencia de actividad física, entre otras variables que serán usadas para determinar una rutina de entrenamiento y/o validar si el usuario es o no apto para la práctica del ciclismo. Como se observa en la figura 5, el sistema se divide en varios módulos de negocio. En la Tabla 1, se resume la descripción de cada uno de los módulos que integra el software.

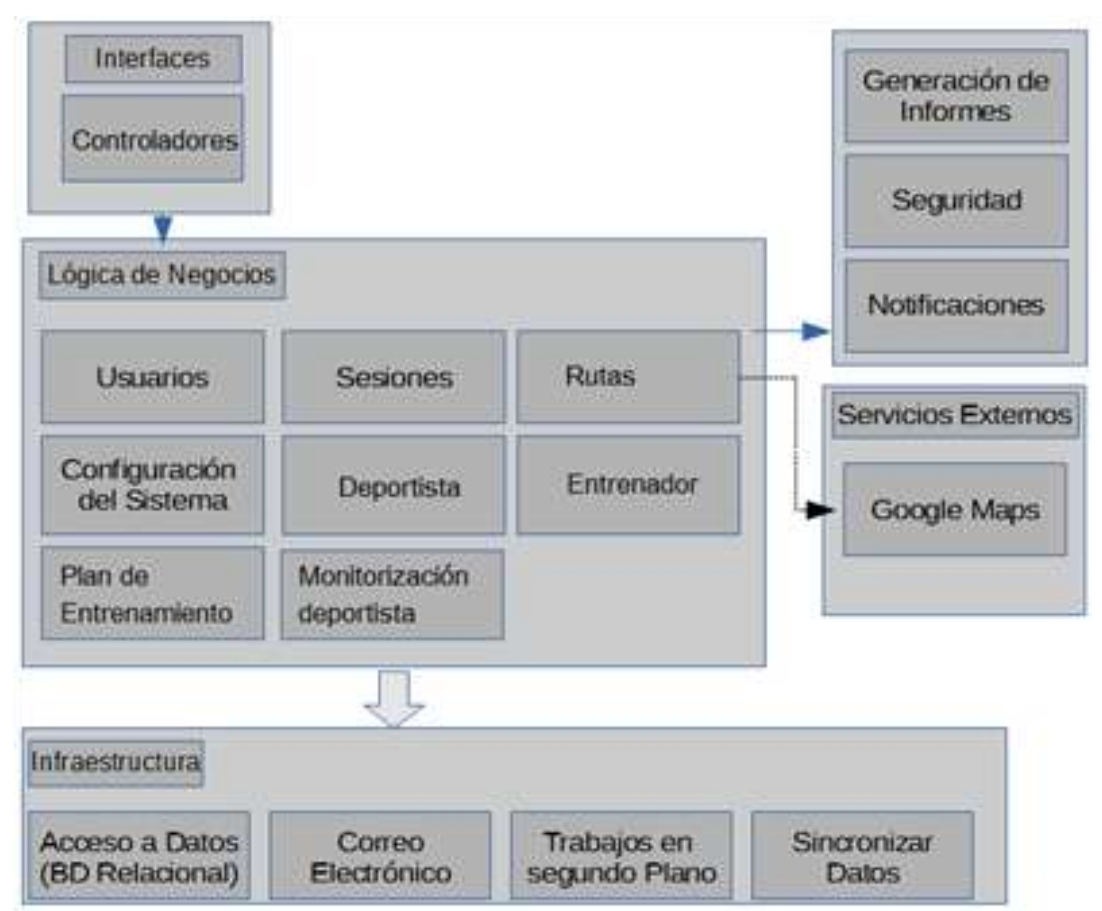

Fig. 5: Modelo de Componentes 
Tabla 1: Módulos de lógica del negocio del sistema

\begin{tabular}{|c|c|}
\hline Módulo & Descripción \\
\hline Usuarios & $\begin{array}{l}\text { En este módulo se realiza la creación, edición y eliminación de usuarios, perfiles y } \\
\text { permisos sobre el software propuesto, con el propósito de tener un control sobre los } \\
\text { módulos que integran la aplicación. }\end{array}$ \\
\hline $\begin{array}{l}\text { Configuración del } \\
\text { Sistema }\end{array}$ & En este módulo se lleva a cabo la parametrización de los procesos y eventos del sistema. \\
\hline $\begin{array}{l}\text { Planes de } \\
\text { Entrenamiento }\end{array}$ & $\begin{array}{l}\text { Este módulo permite al experto o entrenador la creación del plan de entrenamiento } \\
\text { específico para el practicante del deporte, teniendo en cuenta su condición física, estado } \\
\text { de salud y otras variables necesarias para determinar el rango de esfuerzo (mínimo y } \\
\text { máximo) que puede realizar. }\end{array}$ \\
\hline Sesiones & $\begin{array}{l}\text { En este módulo se realiza la administración (creación, edición y eliminación) de las } \\
\text { sesiones de entrenamiento grupal o individual, teniendo en cuenta el plan de } \\
\text { entrenamiento asignado por el entrenador para un perfil de practicante. }\end{array}$ \\
\hline Deportista & $\begin{array}{l}\text { En este módulo se realiza la administración del historial médico deportivo de los } \\
\text { practicantes, y permite visualizar la evolución en rangos de tiempo, posibles lesiones o } \\
\text { problemas presentados. }\end{array}$ \\
\hline $\begin{array}{l}\text { Monitorización del } \\
\text { deportista }\end{array}$ & $\begin{array}{l}\text { En este módulo se realiza el control y seguimiento de los ciclistas en una sesión de } \\
\text { entrenamiento, teniendo en cuenta kilometraje y tiempo de recorrido, calorías quemadas, } \\
\text { entre otros. }\end{array}$ \\
\hline Rutas & $\begin{array}{l}\text { En este módulo se realiza la creación, edición, eliminación de una ruta de entrenamiento a } \\
\text { partir de la captura de datos en tiempo real, así como también permite la simulación del } \\
\text { terreno en una sesión de entrenamiento en ciclismo bajo techo o al aire libre. }\end{array}$ \\
\hline Entrenador & $\begin{array}{l}\text { En este módulo se realiza la creación, edición, eliminación y parametrización de un usuario } \\
\text { entrenador y asignación de responsabilidades de un paciente, planes y sesiones de } \\
\text { entrenamiento. }\end{array}$ \\
\hline
\end{tabular}

De igual manera el software incluye el componente encargado de la recolección de puntos cardinales mediante el uso del GPS del teléfono celular, dicha funcionalidad tiene como objetivo conocer la ubicación del deportista en un lapso de tiempo determinado. Mediante la recolección de estos datos se traza la ruta que se almacenará y posteriormente será la base para una sesión de entrenamiento. Teniendo en cuenta la pendiente, distancia y giros, se identificará la escala de dificultad de cada ruta y esa información servirá para categorizar a los usuarios practicantes en grupos de entrenamiento homogéneos según sus características previamente registradas.

El componente alojado en el teléfono inteligente Android, es una pieza de software que ejecuta tareas en segundo plano para evitar la pérdida de información, realizando una toma de datos cada cierto tiempo sin la necesidad de que la aplicación se encuentre ejecutada por parte del usuario practicante del deporte. Este componente de software integra artefactos, como la interfaz de usuario mediante la cual se configuran datos, entre ellos la identificación única del dispositivo inteligente Android (Imei) y la dirección IP del servidor con el cual se va a realizar la comunicación, también se encuentran los botones asignados a iniciar o detener las actividades en segundo plano (denominado "Servicio"), como se puede observar en la figura 6.

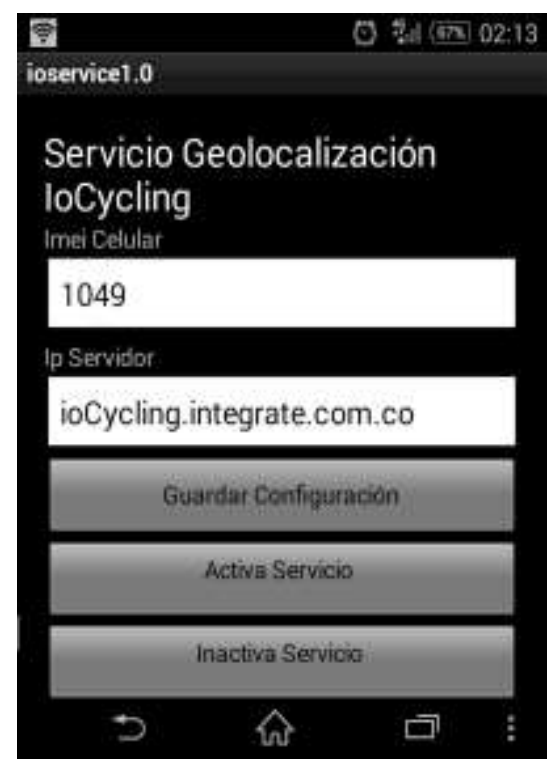

Fig. 6: pantalla Configuración del servicio Android. 
La ejecución de las tareas en segundo plano se realiza a partir de la toma de datos mediante el GPS, presente en el teléfono inteligente. Los tiempos determinados de toma de datos se plantean en lapsos no superiores a 4 segundos, dado que la velocidad alcanzada y la diversidad de terreno de la sesión de entrenamiento pueden variar de un segundo a otro. Los puntos cardinales serán de gran importancia en la creación de rutas de sesiones de entrenamiento, las cuales se realizan por usuarios expertos en la práctica del ciclismo, y finalmente con la colección de puntos ya registradas como una ruta, se usa la herramienta de mapas para trazar los caminos recorridos y dar a conocer las pendientes presentadas, entre otra información que categoriza el nivel de la sesión. Se tiene como componente adicional en la toma de datos, el almacenamiento de información de manera local en el dispositivo Android, como alternativa al momento de presentar inconvenientes en la comunicación al servidor en la nube, por esta razón la información se almacena usando SQLlite y manejando estados de envío y tiempos exactos de toma de datos evitando duplicidad en la información.

\section{RESULTADOS}

En los resultados obtenidos en esta versión del sistema, se da a conocer un muestreo de los datos recolectados en la creación de una ruta de entrenamiento, dichos valores se realizan mediante la toma de información en intervalos de tiempos, por ejemplo de 4 segundos como se observa en la Tabla 2, pudiendo definir un intervalo diferente, dependiendo de la calidad de la señal que ofrece el operador de telefonía móvil en la ruta. Estos valores son almacenados en el servidor central, el cual mediante un algoritmo basado en el servicio de Google Maps, realiza el trazado de la ruta desde el punto inicial hasta el punto final desplegándola en la pantalla del dispositivo móvil, como se observa en la figura 7 , para dar a conocer al usuario practicante la ruta por la cual se sugiere realizar la sesión de entrenamiento.

Tabla 2: Muestra de datos del servicio recolector de puntos de posición global, altura y velocidad.

\begin{tabular}{|c|c|c|c|c|c|c|}
\hline Orden toma de datos & ID Usuario & Longitud ( $(\stackrel{)}{)}$ & Latitud $(\stackrel{\circ}{)})$ & Fecha Lectura & Velocidad $(\mathrm{km} / \mathrm{h})$ & Altura $(m)$ \\
\hline 1 & 1049 & -73.34820 & 5.56072 & 2015-06-25 07:53:31 & 10,012 & 2710 \\
\hline 2 & 1049 & -73.34850 & 5.56046 & 2015-06-25 07:53:35 & 10,512 & 2711 \\
\hline 3 & 1049 & -73.34881 & 5.56023 & 2015-06-25 07:53:39 & 11,761 & 2704 \\
\hline 4 & 1049 & -73.34925 & 5.55915 & 2015-06-25 07:53:42 & 18,257 & 2708 \\
\hline 5 & 1049 & -73.34951 & 5.55852 & 2015-06-25 07:53:43 & 18,007 & 2712 \\
\hline 6 & 1049 & -73.34979 & 5.55802 & 2015-06-25 07:53:47 & 12,510 & 2712 \\
\hline 7 & 1049 & -73.34990 & 5.55770 & 2015-06-25 07:53:51 & 5,5227 & 2715 \\
\hline 8 & 1049 & -73.34994 & 5.55763 & 2015-06-25 07:53:55 & 1,8200 & 2716 \\
\hline 9 & 1049 & -73.34994 & 5.55762 & 2015-06-25 07:53:59 & 0,5 & 2718 \\
\hline 10 & 1049 & -73.34994 & 5.55761 & 2015-06-25 07:54:03 & 0,5 & 2717 \\
\hline
\end{tabular}

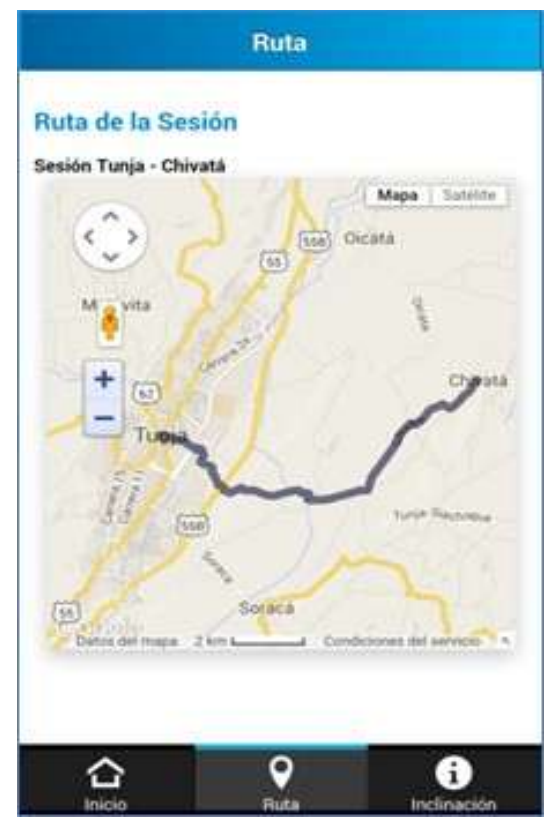

Fig.7: Ruta de la sesión de entrenamiento 
El servidor a partir de los datos almacenados genera la gráfica de elevación de la ruta como se muestra en la figura 8 , con estos datos se realiza la simulación de esfuerzo que se deberá realizar en una sesión de entrenamiento futura, permitiendo la categorización de dificultad de cada una de las ruta programadas, así como la selección de usuarios aptos para cada recorrido.

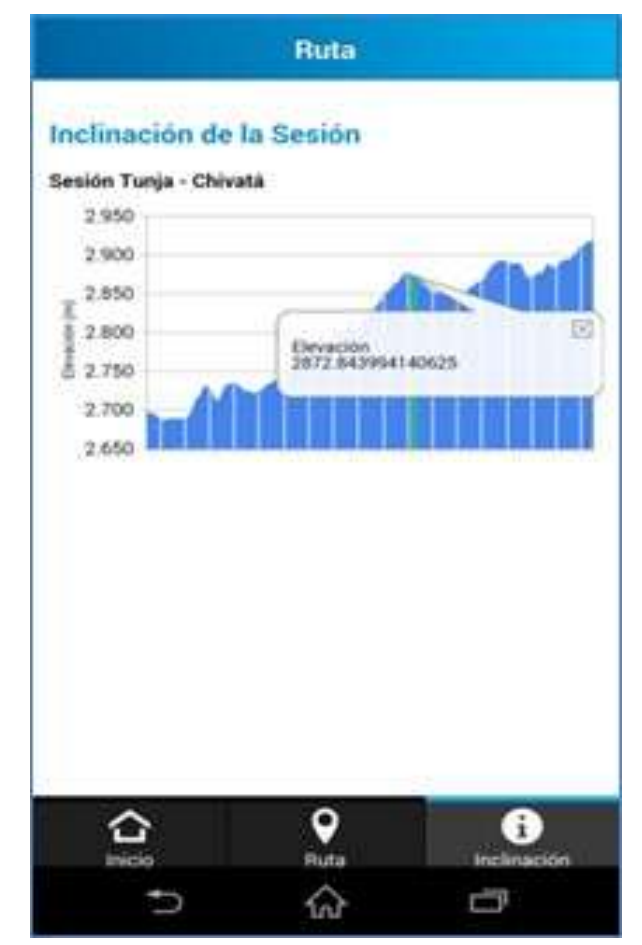

Fig. 8: Inclinación de la sesión de entrenamiento.

\section{CONCLUSIONES}

Aunque existen trabajos relacionados con la temática de esta investigación, es evidente que son altos los costos en lo que un practicante de ciclismo debería incurrir, razón por la cual se desarrolló esta aplicación, que integra los módulos para la administración, control y monitorización de variables físicas del deportista, de tal manera que sea posible determinar el estado físico, rendimiento, progreso en la disciplina, ubicación geográfica y posición dentro de un grupo de practicantes, y de esta manera contribuir en el mejoramiento de la actividad deportiva.

Algunas de las características propias del sistema descrito es que los lapsos de toma de datos no son mayores a 4 segundos para dar más confiabilidad en el trazado de la ruta, y que dichos datos son almacenados de manera local en el dispositivo Android para evitar pérdidas de información y así definir adecuadamente las sesiones de entrenamiento. Además el componente de administración de tareas en segundo plano, evita que la aplicación móvil se cierre por manipulación incorrecta del practicante del deporte, y que se ocasione pérdida de información.

El software desarrollado es una herramienta portable y adaptable, que permite configurar de manera personalizada las diferentes rutas por las que un deportista de ciclismo al aire libre puede entrenar, y a su vez genera insumos para la simulación de rutas dispuestas para practicar ciclismo en modalidad bajo techo.

\section{REFERENCIAS}

Caballé, S. y F. Xhafa. Aplicaciones distribuidas en Java con tecnología RMI, 1era. Edición, 72-76, Delta Publicaciones, Madrid, España, (2007)

Chi-Wai, R.K., T.M. So-Ning, K.L. Wing-Kuen, S.S.-C. Hui, P.W. C.C.-K. Ka-Shun, Wong. Can Mobile Virtual Fitness Apps Replace Human Fitness Trainer?, The 5th International Conference on New Trends in Information Science and Service Science, pp. 56-63.(2011) 
Dora, C. y M. Phillips. Transport, Environment and Health, World Health Organization, Regional Publications, European Series, ISSN 0378-2255, (en línea), 89, 2000.

http://www.euro.who.int/_data/assets/pdf_file/0003/87573/E72015.pdf. Acceso 4 de Septiembre de (2015)

EllisLab Inc. Guía Del Usuario de Codelgniter Versión 2.0.3. Disponible en:

https://ellislab.com/codeigniter/user-guide/installation/downloads.html. Acceso 14 de Septiembre de (2015)

Gaidos, O. F., y I. Santos. Mobile System of Monitoring and Training Cyclists with Smartphone, VI Latin American Conference in Biomedical Engineering CLAIB (2014)

Hintzy, F. y N. Horvais, Non-circular chainring improves aerobic cycling performance in non-cyclists, doi: 10.1080/17461391.2015.1086817, European Journal of Sport Science, 6 p. (2015)

Hirose, S. y Y. Kitamura, Preliminary evaluation of virtual cycling system using google street view, doi: 10.1007/978-3-319-20306-5_6, Lecture Notes in Computer Science (including subseries Lecture Notes in Artificial Intelligence and Lecture Notes in Bioinformatics), 9072, pp. 65-70, (2015)

Kikuchi, T., K. Kobayashi y M. Sugiyama. Development of Virtual Reality Bike with Cylindrical MR Fluid Brake, Proceedings of the IEEE International Conference on Robotics and Biomimetics, pp. 1753 - 1758, (2012)

Lizárraga, M., S. Gallina y M. Zamboni. Servicios En Internet Para Monitoreo Y Control a Distancia Utilizando EDGE / GPRS-GSM, Jornadas de Inf. Ind. - Agroinformática, pp. 359-371 (2008)

Lu, F. y J. Welton. Towards Combating Youth Obesity with a Mobile Fitness Application, Proceedings 17th IEEE International Conference on Wireless, Mobile and Ubiquitous Technology in Education, WMUTE (2012)

Lu, F., K. Turner y B. Murphy. Reducing Adolescent Obesity with a Mobile Fitness Application: Study Results of Youth Age 15 to 17, IEEE 15th International Conference on e-Health Networking, Applications and Services (2013)

Moller, A. y Otros siete autores. GymSkill: A Personal Trainer for Physical Exercises, IEEE International Conference on Pervasive Computing and Communications, PerCom (2012)

Monroy, M. E, J.L. Arciniegas y J. C. Rodríguez. Propuesta Metodológica para Caracterizar y Seleccionar Métodos de Ingeniería Inversa, doi: 10.4067/S0718-07642013000500004, Revista Información tecnológica, (en línea), 24(5), 23-30, (2013)

Nurseitov, N., M. Paulson, R. Reynolds y C. Izurieta. Comparison of JSON and XML Data Interchange Formats: $\quad A$ Case Study, Journal Scenario, pp. 157-162, 2009. http://www.cs.montana.edu/izurieta/pubs/caine2009.pdf. Acceso 14 de Noviembre de (2015)

Unocero. Pioneer Presenta Sistema de Monitoreo Para Ciclistas de Alto Rendimiento http://www.unocero.com/2013/01/11/2013ces-pioneer-presenta-sistema-de-monitoreo-para-ciclistas-de-altorendimiento (2013)

Xie, B. y Q. Wu, Hmm-Based Tri-Training Algorithm In Human Activity Recognition With Smartphone, Proceedings of IEEE 2nd International Conference on Cloud Computing and Intelligent Systems (CCIS), (2012)

Xu, J., X. Nan, V. Ebken, Y. Wang, G.J. Pottie, W.J. Kaiser, Integrated Inertial Sensors and Mobile Computing for Real-Time Cycling Performance Guidance via Pedaling Profile Classification, doi: 10.1109/JBHI.2014.2322871 , IEEE Journal of Biomedical and Health Informatics, (en línea), vol. 19, no. 2 (2015) 